\title{
Demokratie in der europäischen Krise
}

\author{
HAUKE BRUNKHORST
}

Die Gründung der Europäischen Gemeinschaften in Paris 1951 und Rom 1957 geht auf einen politischen Impuls zurück. Alle Staaten, die sich 1951 und 1957 zu den ersten Europäischen Gemeinschaften zusammengeschlossen haben, sind nach 1945 durch neue Verfassungen neu gegründet worden. Die neuen Verfassungen der Gründungsmitglieder orientieren sich nicht nur in ähnlicher Weise an egalitärer Menschenwürde und universellen Menschenrechten, konstituierten die nationale Gesellschaft als soziale Massendemokratie und den von ihnen hervorgebrachten Staat als internationalrechtlich offenen Staat. Sie erklären darüber hinaus auch ihren ausdrücklichen Willen zur europäischen Einigung. Die Gründungsverträge führen deshalb lediglich die Verfassungsprinzipien der Gründungsnationen zu einem höherstufigen constitutional moment zusammen, um die von vornherein supranational organisierten Gemeinschaften als Vereinigungsprojekt eines demokratischen Europa zu begründen. ${ }^{1}$ Damit waren die Europäischen Gemeinschaften durch einen sekundären revolutionären Gründungsakt, der sich direkt aus der verfassungsgebenden Gewalt der neu konstituierten Gründernationen - und nicht nur aus der verfassungsrechtlich

1 Fossum/Menéndez 2011. 
unverbindlichen, völkerrechtlichen Verbindung ihrer Staaten - herleitete, legitimiert.

Mit der Rückbindung der Unionsverträge an die verfassungsgebende Gewalt der Völker sind von vornherein die Völker und nicht ihre Staaten, durch deren Organe sie in den Vertragsverhandlungen vertreten wurden, in einem viel stärkeren Sinn die „Herren der Verträge“ als in einem gewöhnlichen intergouvernementalen Vertrag. ${ }^{2}$ Sie konstituieren sich im Vertragsabschluss nicht nur zu einem Staatenbund oder Staatenverbund, sondern zu einem Völkerbund, also nicht nur als Gemeinschaft demokratischer Staaten, sondern als autonome demokratische Gemeinschaft mit einer eigenen parlamentarischen Versammlung (Gemeinsame Versammlung von 1952), aus der später das Parlament hervorging. Die Gemeinschaftsverträge waren, weil sie durch die verfassungsgebende Gewalt der Gründungsvölker direkt legitimiert waren, unmittelbarer Ausdruck des jeweils nationalen Verfassungsrechts, und die nationalen Verfassungen deshalb mit Vertragsabschluss zu Teilverfassungen der Gemeinschaft geworden. Das entsprach im Übrigen nicht nur dem Pathos und Inhalt der Präambel schon des Pariser Vertrags, sondern auch der dort vollzogenen Vergemeinschaftung des Teils der nationalen Wirtschaft, ohne den dem einzelnen Staat keine nach Vorkriegsverständnis souveräne Außenpolitik mehr möglich war - waren doch Kohle und Stahl 1951 noch unverzichtbar, um selbständig Kriege zu führen. Zu jener Zeit war die Angst vor einer Fortsetzung des Zweiten Weltkriegs noch überall in Europa spürbar, und man wusste noch, was das bedeutet. Nicht nur die gemeinsame Wirtschafts-, auch die gemeinsame Außenpolitik beginnt 1951.

In zwei Präzedenzfällen (Van Gent und Costa) hat der Europäische Gerichtshof dann 1963 und 1964 in einer stark teleologischen Interpre-

2 Vgl. dazu auch meine Aufsätze zum Legitimationsproblem der Europäischen Union: Brunkhorst 2012. Ähnlich im Anschluss an Ulrich Thiele und Peter Niesen jetzt auch: Habermas 2011, 39 f., Fn. 48, 67. Vor allem Thiele hat den systematischen Unterschied zwischen Völker- und Staatenbund bei Kant scharf herausgearbeitet; Thiele 2011, 175-196. 
tation der Verträge von Rom die Wirtschaftsgemeinschaft als Gemeinschaft verstanden, die nicht nur durch die beteiligten Nationen (Staatengemeinschaft), sondern auch durch eine autonome europäische Bürgerschaft konstituiert wird. Indem die Verträge subjektive Rechte erzeugt haben, die den einzelnen Individuen nicht als Angehörigen ihrer jeweiligen Völker zukommen, sondern nur in ihrer Rolle als Bürger Europas, hat sich - ganz analog zu der im Amerikanischen Verfassungsrecht seit Marbury vs. Madison üblichen Rede von der geteilten Souveränität - die ursprüngliche verfassungsgebende Gewalt der Völker Europas in nationale Völker und Europäische Bürgerschaft geteilt. ${ }^{3}$ Der seinerzeitige Generalanwalt des EuGH, Miguel Poiares Maduro spricht in diesem Zusammenhang vom ,Wunder der Unionsbürgerschaft“", dass sie einerseits „die Bindung an unsere Staaten“ verstärke, „soweit wir deshalb Unionsbürger sind, weil wir Angehörige unserer Staaten sind“, uns andererseits aber ,zugleich“ „,von ihnen“ „emanzipiert", ,soweit wir nunmehr Bürger über unsere Staaten hinaus sind““.

Das nationale bildet heute mit dem europäischen Verfassungsrecht ein dichtes Kontinuum, das viele Unterschiede, aber keinen Dualismus von nationalem und internationalem Recht mehr kennt (wie ihn das BVerfG in seinen Europaurteilen regelmäßig unterstellt). ${ }^{5}$ Unspektakuläre Alltagsroutinen, juristischer und politischer Inkrementalismus haben schließlich ein komplexes System gesamteuropäischer Gewaltenteilung geschaffen, in das die nationalen Staatsgewalten mittlerweile fast lückenlos zu einem einzigen, großen Organismus integriert sind.

3 Habermas 2011; s. a. schon: Schönberger 2005.

4 Schlussanträge Maduro v. 30. 9. 2009, zit. n. Kemmerer 2010, 216 f., meine Hervorhebung.

5 Zur wichtigen Unterscheidung von „Unterscheidung“ und „Dualismus“ vgl. Brandom 2000, S. 856 ff., insbesondere S. 864-866. Zum Europasyndrom des Verfassungsgerichts: van Ooyen 2010. Die Dualismuskritik geht im Staatsrecht auf Kelsen zurïck, auch wenn das meist nicht gesehen wurde, weil Kelsen als Neukantianer ad acta gelegt wurde. Das revidiert: Brunkhorst 2011, 496-512. 
Dieser Organismus ist heute schon, wie das tschechische Verfassungsgericht in einem bahnbrechenden Urteil zum Lissabonner Vertrag festgestellt hat, ein einheitliches System demokratischer Legitimation. ${ }^{6}$ Insofern ist dem Hegelianismus von Habermas zuzustimmen, dass in den Verträgen an sich schon alles enthalten ist, um eine poststaatliche, demokratische Regierung Europas zu begründen. Man muss das demokratische Europa nur durch immanente Kritik aus der bestehenden Rechtsordnung herausholen, explizit machen und dann auch noch zum Sprechen bringen. ${ }^{7}$

Aber das genau ist das Problem. Europa ist als Union zwar bereits demokratisch verfasst. Aber das weiß keiner.

Das Problem ist nicht die inkrementalistische Berufspolitik als solche, der Lobbyist im Hinterzimmer, die (vorgeblich) riesige Bürokratie, die technische Apparatur, der öffentlich zugängliche, aber unlesbare Gesetzestext-500-seitige Verfassungsverträge voller juristischer Tücken und Fallstricke, die nur noch Spezialisten mit Sonderausrüstung erkennen können. Das Problem ist die Reduktion von Politik auf Technik unter Umgehung, Ausschaltung und Manipulation des öffentlichen Meinungskampfes und öffentlicher Willensbildung. Christoph Möllers hat diese Form entpolitisierter Politik treffend als bypassing der öffentlichen Macht des Volkes und seiner Organe durch Netzwerke informeller Herrschaft charakterisiert. ${ }^{8}$ Durch die Reduktion von Politik auf Techniken des Machterhalts wird die demokratische Form der europäischen Integration von vornherein von ihrem demokratischen Inhalt abgetrennt. Kein Wunder, dass dieser sich in sein Gegenteil verkehrt, auf Immigranten eindrischt, die dumpfesten Vorurteile gegen Griechen und Türken, Sinti und Roma mobilisiert und unter Leitung

6 Ley 2010. Zum prozeduralen Demokratiebegriff: Habermas 1992; zum „synthetic constitutionalism“: Fossum/Menéndez 2011.

7 Habermas 2011.

8 Möllers 2005, 351-389; Möllers 2003. Zur Akkumulation informeller Macht in flexiblen, weit verstreuten und rasch wechselnden Zentren s. auch Hardt/Negri 2003; Prien 2010; Fischer-Lescano/Teubner 2006. 
populistischer Führer gegen Europa und die Demokratie zu Felde zieht. Das Parlament kann gehen, Adel und Banken sollen's richten. Als noch alle Welt vom deutschen Verteidigungsminister Guttenberg schwärmte, motivierte dessen hoher Adelstitel sogleich die deutschen Talkshows zur Frage „Brauchen wir wieder einen König?“ Und als Lehmann Brothers zusammenbrach und auch Herr Ackermann von der Deutschen Bank nicht mehr ein noch aus wusste, fragten sich die Talkshow-Schönheiten, nachdem sie gerade zuvor die Politiker demontiert und durch braungebrannte Muskelschränke aus der mittelständischen Exportbranche ersetzt hatten, irritiert: „Auf wen sollen wir denn jetzt noch hören?".

Kurz und schlecht: Inkrementalistische Politik hat den paradoxen Effekt, dass die Anti-Europa-Parteien zur öffentlich sichtbarsten und stärksten Kraft der Europäischen Union werden und schon geworden sind. Sie repräsentieren Europas Einheit unter der Parole: „Schluss mit Europa!“

Technische Politik sollte man indes nicht verachten. Sie hat einen großen Vorteil. Ihre Ergebnisse sind erwartbar. Sie schafft Sicherheit, sie reduziert Komplexität - und zwar im legitimen Interesse aller Bürger. Technische Politik ist darauf programmiert, dass Überraschungen ebenso ausbleiben wie in der Flugzeug- oder Agrartechnik, die das Fliegen oder die Schweinezucht zuverlässig und erwartbar machen. Deshalb ist das bypassing der flatterhaften öffentlichen Meinung, der spontanen öffentlichen Selbstbestimmung und der verfassungstreuen öffentlichen Kontrolle das A und O technokratischer Exekutivpolitik. Und wenn es hart auf hart kommt, sagt sie, Not kennt kein Gebot. ${ }^{9}$

Technische Politik ist unvermeidlich, aber strukturell undemokratisch, in sich nicht demokratisierbar. Der Bologna-Prozess war exemplarisch. Die größte und tiefgreifendste Hochschulreform, die Europa je erlebt hat und die jene des 19. Jahrhunderts weit in ihren Schatten stellte, hat sich in den Massendemokratien des 21. Jahrhunderts, in dem sie nicht mehr ein Prozent, sondern dreißig bis vierzig Prozent der Bevöl-

9 Böckenförde 2012. 
kerung betrifft, fast geräuschlos vollzogen. Auf der Basis eines rechtlich gänzlich unverbindlichen Protokolls hatten sich in trauter Private Public Partnership die nationalen Exekutivspitzen der Wissenschaftsverwaltung zusammen mit einem Vertreter der Zivilgesellschaft, dem Abgesandten des Bertelsmann-Konzerns, eines schönen Tages in Bologna geeinigt, die Universitäten eins zu eins dem Vorbild der globalen Finanzindustrie nachzubilden: Im Zentrum ein paar Exzellenzblasen, und in der Peripherie wüste Leere. In der Parlamentsvorlage zu SchleswigHolsteins Hochschulgesetz liest man dann verwundert, der Bolognaprozess müsse umgesetzt werden. Parlamentarismus als selbst verschuldete Unmündigkeit. ${ }^{10}$

Irgendwann jedoch stößt der durchaus effektive Mix aus technischer Politik und juridifizierter Konstitutionalisierung an die Schranken seines eigenen, verfassungsrechtlichen Bauplans. Dann passiert, was der Technik in aller Regel nicht passiert, dass sie nicht funktioniert und die Kontrolle über das Kaiserreich, die arabischen Massen, das Flugzeug oder die Schweinegrippe verliert - dann freilich, um es mit Luhmann zu sagen, mit Kaskaden von Nebenfolgen.

Mit fortschreitender Komplettierung des Konstitutionalisierungsprozesses tritt der inkrementalistische Funktionalismus, dessen Hauptakteure politische Eliten und juristische Experten sind, in einen immer schärferen Gegensatz zum - und das ist hier meine These - gleichzeitig wachsenden emanzipatorischen Gehalt des Europäischen Rechts. Technokratische Herrschaft, da sie sich als Herrschaft des Rechts und durch Recht vollziehen muss, kann das nur, wenn gleichzeitig der emanzipatorische Gehalt der von ihr beherrschten Rechtsordnung steigt. Deshalb kann technokratisch neutralisierte Politik über Nacht in eine ernsthafte Legitimationskrise Europas umschlagen. Es könnte mit der Union dann so schnell zuende gehen wie mit dem Herrn Mubarak, wurde doch auch der mächtige Belsazar noch „in selbiger Nacht/Von seinen Knechten umgebracht“" (Heine).

10 Brunkhorst 2006, 1-6. 
Bevor ich auf die gegenwärtige Krise komme, möchte ich skizzieren, wie es zum Triumph technischer Politik und zur Verdrängung des Politischen in Europa kommen konnte. Meines Erachtens war schon der Anfang mit der Wirtschaftsverfassung der Sündenfall, und er pflanzt sich, wie im Dogma der Erbsünde, im Prozess der immer enger werdenden Union von Entwicklungsstufe zu Entwicklungsstufe fort.

Die Evolution des europäischen Verfassungsrechts lässt sich in vier Stufen rekonstruieren.

Auf Stufe I werden infolge der rechtlich ermöglichten, funktionalen Ausdifferenzierung eines Europäischen Wirtschaftssystems Recht und Wirtschaft zu einer Funktionsverfassung verkoppelt. Legitimation wird auf output-Legitimation rediziert und von demokratischer Legitimation entkoppelt.

Die Wirtschaftsverfassung Europas war von Anfang an hegemonial strukturiert und ist heute, das wird in der Weltwirtschafts- und EuroKrise der unmittelbaren Gegenwart auch öffentlich sichtbar, mehr denn je deutscher Großraum, der im Zentrum einen Aktiensturm entfacht, die Inflationsrate neurotisch flach hält und die Peripherie, in der die Banken des deutschen Hegemon gewaltige Gewinne einfahren (oder bis vor kurzem eingefahren haben), in eine Flaute unabsehbarer Deflation treibt. ${ }^{11}$

Gegen den Widerstand Frankreichs wurde 1957 die freie Marktwirtschaft im Art. 2 EEC zur „Grundentscheidung“ (Carl Schmitt) der europäischen Verfassungsgeschichte. Damit hatte sich die von der USRegierung massiv unterstützte und von Müller-Armack (NSDAPMitglied von 1933-1945) maßgeblich formulierte deutsche Position in den Vertragsverhandlungen gegen die stärker Keynesianische Ausrichtung Frankreichs und der anderen Staaten der Gemeinschaft durchgesetzt. $^{12}$

11 Joerges 2003, 167-191.

12 Wegmann 2010, hier 94, 99 f., 102 f.; zur Durchsetzung des Ordo-/Neoliberalismus der Deutschen Bundesbank im Verfassungsstreit um Stabilitätsund Geldpolititk der Zentralbank, s.: Gaitanides 2005, 550-558, hier: 553 f. 
Der Ordoliberalismus war seit den späten 1920er Jahren ein konterrevolutionäres, ebenso gegen den Marxismus und die Sozialdemokratie wie gegen Keynes gerichtetes Projekt. Das Programm einer Wirtschaftsverfassung hat der Ordoliberalismus indes von der Linken übernommen, oder, wie Kaarlo Tuori treffend schreibt, „rather hi-jacked it“. ${ }^{13}$ Am Ende des Ersten Weltkriegs war die Idee einer umfassenden Wirtschafts- und Sozialverfassung von Hugo Sinzheimer und seinen Schülern in Umlauf gebracht und zumindest teilweise (durch Mitwirkung Sinzheimers) der Weimarer Verfassung implementiert worden. Die ordoliberale Wirtschaftsverfassung war eine stark verwässerte Version der sozialistischen Ideen der Frankfurter Sinzheimer-Schule, deren „konterrevolutionäre“ Absicht darin bestand, den Sozialismus durch eine politisch geordnete liberale Marktwirtschaft zurückzudrängen, die dann unter dem ideologischen Label der ,sozialen Marktwirtschaft" von Ludwig Erhard als Wunderwaffe des Nachkriegsbooms in Umlauf gebracht wurde: Rheinischer Kapitalismus.

Legte der Ordoliberalismus noch auf einen breiten Wettbewerb wert, der eine nivellierte Mittelstandsgesellschaft durch eine sozial zumindest minimal abgefederte Marktwirtschaft gewährleisten sollte, so setzt der global denkende Chicagoer Neoliberalismus (Milton Friedman) bereits ganz auf die marktbeherrschende Organisationsmacht weniger großer, weltweit operierender Konzerne, die weitgehend an die Stelle der Staatsmacht und ihrer Sozial- und Wohlfahrtspolitik treten sollen und mittlerweile getreten sind. Die allgemeine Wohlfahrt wird in Konsumentenwohlfahrt umdefiniert und - in einem kühnen Schluss von Konsument auf Aktionär - in steigenden oder sinkenden Aktienkursen direkt gemessen. Die Aktionärsgewinne wurden zum Maß aller Dinge. Das neoliberale Programm war der Globalisierung der Märkte, Wertsphären und Funktionssysteme, die seit den 1960er Jahren rasch an Fahrt gewann, weit besser angepasst als ihre Keynesianischen, sozialdemokratischen oder gar sozialistischen Konkurrenten, die allesamt auf den Verfassungsrahmen des nationalen Staats angewiesen blieben

13 Vgl. Tuori 2010, 3-30, hier: 16. 
oder sich durch die letzte Form der klassischen Imperiumsbildung (Sowjetunion) der Weltgesellschaft zu verweigern suchten. Keynesianismus und die mit ihm fast ubiquitär gewordene Sozialdemokratie Nixon sagte 1970: „We are all Keynesians“ - haben den sozialstaatlich eingebetteten Kapitalismus geschaffen, aber sie hatten - anders als die Neoliberalen - kein Programm für die Globalisierung, noch nicht mal eins für Europa, sieht man einmal von Jaques Delors ab. Das ist das historische Wahrheitsmoment des Neoliberalismus, das ihn zur ubiquitären Episteme gemacht hat - eine Ideologie im klassischen Sinn notwendig falschen Bewusstseins.

Das missing link zwischen Ordo- und Neoliberalismus ist die vom Staat abgelöste, im Sinne Ipsens technisch neutralisierte Wirtschaftsverfassung. Sie schließt eine politische Verfassung jenseits des Nationalstaats kategorial aus. Die politische Verfassung muss staatlich bleiben! Das ist der kategorische Imperativ, der Ordo- und Neoliberale eint - so verschieden sie sonst sein mögen.

Mit der politischen Verfassung bleibt die politische Interventionsund Regulierungsmacht weitgehend auf den Nationalstaat beschränkt, während die vom Staat entkoppelte Wirtschaftsverfassung die Globalisierung der Konzern- und Bankenmacht rechtlich stabilisieren soll. Die Macht der verstaatlichten politischen Verfassung über die Märkte verwandelte sich unter der Obhut der in Europa und darüber hinaus global (GATT, WTO, IMF, World Bank) entstaatlichten Wirtschaftsverfassung in Ohnmacht zurück und die state embedded markets des keynesianischen Zeitalters in market embedded states. In der Europäischen Rechtsprechung markieren die Dassonville-Entscheidung 1974 und die Cassis de Dijon-Entscheidung des EUGH von 1979 die Wende zur einseitigen Dominanz der vier ökonomischen Freiheiten. ${ }^{14}$ Menéndez

14 Vgl. Fisahn 2011, 48-60, hier: $50 \mathrm{f}$. Kritisch zum possessiv individualistischen Paradigma der Integrationspolitik und seinem latenten Autoritarismus: Somek 2008; aufschlussreiche Fallstudie zur Emergenz des neoliberalen Paradigmas: Buckel/Oberndorfer 2009, 277-296. 
und Fossum sprechen treffend von einer Emanzipation der ökonomischen Freiheiten vom nationalen Verfassungsrecht. ${ }^{15}$

Aber schon mit der Grundentscheidung der Wirtschaftsverfassung war der Weg in einen europäischen Sozialstaat, ohne den das demokratische Projekt einer politischen Verfassung unvollendet bleiben muss, wirksam blockiert worden. ${ }^{16}$ Wie Ipsen richtig gesehen hat - und Majone und Moravzik sind ihm darin gefolgt -, bedarf es für eine Wirtschaftsverfassung nur einer technokratischen Funktionselite, die sie verwaltet, aber keiner demokratischen Bürgerschaft.

Die soziale Evolution ging dann aber Zug um Zug darüber hinaus, indem sie emanzipatorischen Gehalt Europäischen Rechts mit jedem Integrationsschritt stärkte. Dabei wurde jedoch die Hegemonie der technokratisch-expertokratischen Regimeform zu keinem Zeitpunkt ernsthaft in Frage gestellt. Auf allen Entwicklungsstufen wurde das Politische, wurde die Politik der Demokratie erfolgreich verdrängt und in den Nationalstaat, der allein immer weniger zu entscheiden hatte, zurückgedrängt.

Erst kam auf Stufe II seit den frühen 1960er Jahren - lange vor den Pässen - die rechtsstaatliche, privatautonome Konstitution einer europäischen Bürgergesellschaft. Technisch gesprochen handelte es sich um die strukturelle Kopplung von Recht und Rechten durch gerichtlich erzeugtes und fortgebildetes Recht. Die Evolution der Rechtsstaatsverfassung Europas reagiert auf die beginnende Europäisierung des Rechtssystems. Mit rasant wachsendem Normbedarf wuchs die Zahl der Konflikte, und die Gerichte haben daraus die Rechtsstaatsverfassung Europas gemacht, bestehend aus der Europäischen, die die Weichen gestellt hat und den vielen Nationalen, die tausende von Fällen des Typs „Hauptzollamt Bielefeld vs. Molkereibetrieb Sennestadt“ zu entscheiden hatten. ${ }^{17}$ Dadurch wurde der emanzipatorische Gehalt des

15 Fossum/Menéndez 2011, 115 f.

16 Holmes 2012.

17 Vgl. Alter 1996, 458-487; Alter 1998, 121-147; Alter/Meunier-Aitsahalia 1994, 535-561; Hitzel-Cassagnes 2011. 
Europarechts (Privatautonomie) zwar gesteigert, aber nur um den Preis der zurückbleibenden politischen Autonomie, die durch die wachsende Macht technokratischer Exekutivpolitik kompensiert wurde.

Das dadurch immer offensichtlicher werdende Demokratieproblem der Union wurde dann durch die Evolution der politischen Verfassung Europas seit Einführung des allgemeinen und direkten Wahlrechts zum Europäischen Parlament 1979 gelöst. Das ist dann Stufe III, die durch strukturelle Kopplung von Recht und Politik erklommen wird und auf die rasante Ausdifferenzierung des europäischen politischen Systems in den 1970er und 80er Jahren reagiert. Das formelle Demokratiedefizit ist durch die stetig wachsende informelle Macht eines working- und law-shaping-parliaments nach dem Modell des US-Kongresses und die Einführung des parlamentarischen Gesetzgebungsverfahrens im Lissabon-Vertrag weitgehend behoben. ${ }^{18}$

Europa hat heute eine politische Verfassung. Die Verfassung ist demokratisch. Aber keiner geht hin, und jeder schreibt das Gegenteil. Auch das BVerfG verweigert dem EU-Parlament trotzig und wider besseren Wissens seinen Segen. ${ }^{19}$

Der emanzipatorische Anspruch der politischen Verfassung, die demokratische Selbstbestimmung der europäischen Bürgerschaft zu gewährleisten, wird nämlich vom techno- und expertokratischen Inkrementalismus noch in der Stunde seiner Geburt, wenn nicht vernichtet, so doch zur Latenz verurteilt. Die strukturelle Kopplung von Politik und Recht stabilisiert nicht nur die demokratischen Institutionen wie das Parlament, sondern auch den außerparlamentarischen Machtzuwachs der europäisch vereinigten Exekutiven. Gestaltungsmacht zu erhalten und zu akkumulieren ist die Funktion der Exekutiven, aber nicht ihre normative Bestimmung. Schon seit geraumer Zeit lässt sich die Entstehung eines kollektiven Bonapartismus - Habermas spricht heute mit Colin Crouch, Stefan Oeter und Phillip Dann von ,postdemokratischem“ „Exekutivföderalismus“ - in der rechtlich kaum gebundenen,

18 Dann 2002, Jean-Monnet working paper 5; Bast 2010, 173-180.

19 Schönberger 2009; Halberstam/Möllers 2009; von Bogdandy 2010, 340-350. 
dafür aber umso mächtigeren Gestalt des Europäischen Rats und einer Vielzahl rein exekutiver Sonderregimes beobachten, die Europa seit EURATOM begleiten. $^{20}$

Das ist beim Parlament selbst nicht anders. Auch die wachsende Parlamentsmacht blüht nur im Verborgenen. Der Preis für seinen Machtzuwachs besteht in seiner öffentlichen Delegitimierung. Der Abgrund zwischen wachsender parlamentarischer Gesetzgebungsmacht und - gemessen an der öffentlichen Erkennbarkeit der Alternativen, die zur Wahl stehen - sinkender demokratischer Legitimation wird von Wahl zu Wahl größer. Da nützt auch die Erfindung schöner neuer Namen, deren Liste von der ,guten Regierung“ (good governance) über „deliberative“ und ,auditive“ Demokratie bis hin zur (rätedemokratischen) „Komitologie“ reicht, nichts, wenn damit ohnehin nur good governance without democratic government, inklusive Deliberation ohne egalitäre Dezision, Anhörung ohne Partizipation - oder, wie einst in der Sowjetunion - Rätedemokratie ohne Volk gemeint ist. Kein Wunder, dass die Bürger, wenn sie in Referenden einmal selbst die Wahl haben, den Verfassungsvertrag ablehnen und den Lissabonner Vertrag erst in einem zweiten, für die Demokratie demütigenden Wahlgang, der den Iren mit Zuckerbrot und Peitsche aufgenötigt wurde, akzeptieren. Europas Demokratie bleibt, um mit Susan Marks zu sprechen, low intense democracy - ganz nach dem Geschmack der Hauptaktionäre, der großen Banken und ihrer smarten Chefökonomen. ${ }^{21}$

An die Stelle der Antinomie von subjektiven Rechten und Demokratie tritt auf der Stufe der politischen Verfassung eine neue Antinomie: die Antinomie von öffentlichkeitsfernem Parlamentarismus und demokratischer Öffentlichkeit. In diesem und nur in diesem - freilich entscheidenden - Punkt können die Parlamente in Paris, Brüssel, Berlin oder Madrid heute noch mehr demokratische Legitimation für sich verbuchen als das Parlament in Straßburg (aber die in Italien; Irland. Portugal und Griechenland schon nicht mehr).

20 Brunkhorst 2007, 12-29.

21 Marks 2000. 
Fragt sich nur, ob die bis heute latent gehaltene Legitimationskrise der Union auch dann noch latent gehalten werden kann, wenn die Finanzwirtschaft zusammenbricht, die politische Krise des Euro manifest wird und die Union auf die technokratisch knapp gehaltene Ressource Solidarität zurückgreifen muss, um fortzubestehen. Alles, was wir derzeit erleben, spricht dagegen. Die Wirtschafts-, Finanz- und Eurokrise machen die symptomatische Wahrheit (Zizek) Europas jedermann sichtbar. Die auf Integration, Solidarität und Demokratie ausgestellten Schecks der politisch herrschenden Klasse sind nur noch durch technische Output-Legitimation gedeckt. Werden sie in der demokratischen Münze der Input-Legitimation eingefordert, müssen sie mit einem lauten Knall platzen. Das würde wenigstens dem so lange erfolgreichen bypassing und silencing der öffentlichen Meinung ein plötzliches, unsanftes, aber auch befreiendes Ende bereiten, und dann könnte Europas Parlament vielleicht doch noch einmal die Funktion erfüllen, die Marx einst den nationalen Parlamenten, die der Vereinigung globaler Konzern- und Exekutivmacht heute hilflos gegenüberstehen, zugedacht hatte, als er das parlamentarische Regime das „Régime der Unruhe“ nannte, „das nach dem Ausdrucke eines ihrer Redner im Kampf und durch den Kampf [...] lebt, das [...] von der Diskussion [lebt, das] jedes Interesse, jede gesellschaftliche Einrichtung [...] in allgemeine Gedanken verwandelt, [in dem] der Rednerkampf auf der Tribüne [...] den Kampf der Preßbengel hervor[ruft], der debattirende Klub im Parlament [...] sich nothwendig durch debattirende Klubs in den Salons und in den Kneipen [ergänzt], die Repräsentanten, die beständig an die Volksmeinung appelliren, [...] die Volksmeinung [berechtigen] in Petitionen ihre wirkliche Meinung zu sagen. Das parlamentarische Régime überläßt alles der Entscheidung der Majorität, wie sollen die großen Majoritäten jenseits des Parlaments nicht entscheiden wollen? Wenn ihr auf dem Gipfel des Staates die Geige streicht, was Andres erwarten, als daß die drunten tanzen. “22

22 Marx 1985, 135 f. 
Nun aber scheint die Euro-Krise Europa bei Strafe des Untergangs zu nötigen, zu einer wirklichen Demokratie, und das heißt eben auch: in eine Umverteilungsgemeinschaft $\mathrm{zu}$ mutieren. Denn ohne rough equality (Collin Crouch) keine moderne Massendemokratie. Wir stehen an der Schwelle zu Stufe IV der Konstitutionalisierung Europas, der strukturellen Kopplung des Rechts mit den Sozial-, Wohlfahrts- und Bildungssystemen, die der beginnenden Europäisierung dieser Systeme (Anti-Diskriminierungsnormen, Bologna) allmählich nachwächst und 2008 von der Weltwirtschafts- und Finanzkrise abrupt auf die Tagesordnung gesetzt wurde.

Die Krise, der große Vereinfacher, hat den Staat noch einmal als starken Retter erscheinen lassen, um ihn schon im nächsten Augenblick im letzten Hemd stehen zu lassen. „Noch so ein Sieg, und wir sind verloren“, wie es Wolfgang Streeck formulierte. ${ }^{23}$

Um es in der einfachen Sprache der Krise zu sagen: Der mächtige und reiche, nationale Wohlfahrtsstaat der westlichen Hemisphäre hatte grundsätzlich zwei Möglichkeiten, mit der marktwirtschaftlich (und offensichtlich auch planwirtschaftlich) unaufhebbaren Krisentendenz des Kapitals fertig zu werden. Er kann Milliarden in die Wirtschaft pumpen, und davon hatte er bis vor kurzem mehr als jeder andere global economic player. Oder er kann, statt dem Kapital mit dem Zuckerbrot seiner fast endlosen Kreditwürdigkeit zu winken, zur Peitsche des Gesetzes greifen und kostenfrei in die Wirtschaft intervenieren. Er kann die Geldströme regulieren, abschöpfen, umlenken. Er kann Banken zerstückeln, verstaatlichen, an eine enge Kette aus Gesetz und Verordnung legen. Er kann das Regime des Kapitals dem Regime der Staatsmacht unterwerfen. Er kann dem systemisch selbstsüchtigen Kapital (das hat nichts mit Gier zu tun) das allgemeine Interesse aufnötigen, aber nur, wenn der Staat die Wahl hat, zu zahlen oder zu zerstückeln.

Genau diese Wahl hat und hatte er in der gegenwärtig anhaltenden Krise nicht mehr. Regulieren, Zerstückeln, Verstaatlichen, Anreizen, Anketten, Abschöpfen geht in den Größendimensionen einer funktio-

23 Streek 2010. 
nal differenzierten und systemisch geschlossenen Weltwirtschaft nur noch durch kontinental und global koordiniertes, kooperatives Handeln.

Ohne Alternativen aber kann man nicht planmäßig handeln. Die den staatlichen Geldsegen lenkende und begleitende Peitsche des Gesetzes trifft das Kapital nicht mehr, sondern nur noch den Wind, der um die Erde fegt - so wie einst Xerxes, in einer ähnlichen Zwickmühle, das Meer auspeitschen ließ. Es hat ihm so wenig genützt wie Präsident Obama oder Kanzlerin Merkel das leere Gerede von globaler Regulierung.

Warum ist der Neoliberalismus nicht tot? Vielleicht tatsächlich, wie Colin Crouch meint, weil er - und das war das Wahrheitsmoment in den Theorien Friedmans und der Chicago School - die Gunst der Stunde genutzt und eine so gewaltige, globale Konzern- und Bankenmacht aufgebaut hat, wie sie in der Geschichte noch nie gesehen wurde, während der Staat es nach dreißig Jahren Neoliberalismus, in denen aus state embedded markets market embedded states wurden, verlernt hat, ohne die Krücken von Private Public Partnerships und New Public Management, ohne den Rat der Deutschen Bank, der City of London, der Wall-Street-Giganten, ohne die Unsummen an Beratungsgeldern, die aus den Gewinnen der Versicherungsgesellschaften in die Taschen der Abgeordneten fließen, ohne die kleinen, schäbigen Kreditgeschenke und Upgradings, ohne die dauernde Finanzierung der Nachfrage durch die private Überschuldung verarmter Mittelschichten - Colin Crouch spricht treffend vom ,,privatisierten Keynesianismus“ -, ohne die Überschwemmung des Landes mit faulen Immobilienkrediten überhaupt zu gehen und zu handeln. ${ }^{24}$

Was man verlernt hat ist weg. Und jetzt ist das Geld auch noch weg.

Wenn der Staat nicht mehr die Wahl hat zwischen Zuckerbrot und Peitsche, hat er keine Wahl mehr und muss zahlen. Er ist erpressbar geworden, und er ist erpresst worden, und natürlich hat er gezahlt und

24 Crouch 2011, 170; vgl. a. Judt 2010. 
immer wieder gezahlt und sein letztes Geld, unsere schönen Steuergroschen, dahin gegeben, wo es von ihm verlangt wurde. Die Regierung konnte auch gar nichts anderes mehr tun. Die self-fullfilling prophecy von Margaret Thatcher ist wahr geworden: There is no alternative.

Oder doch? Habermas und Böckenförde sind sich jedenfalls in diesem einen Punkt einig, dass „die jetzige Krise“, wie Böckenförde schreibt, ,auch eine große Chance für Europa“ ist: „Die bisherigen Vertragsregelungen, faktisch bereits überrollt, können nicht unverändert bleiben [...]. Die verschiedenen Optionen müssen offen diskutiert [...] werden. Will man den Euro stabilisieren und stärken [...], muss man entschiedene Schritte hin zu einer politischen Union gehen. Das schließt Souveränitätsverzichte der Mitgliedstaaten und einen Europäischen Finanzausgleich ein." Habermas verweist hier auf den Art. 106 GG zum Länderfinanzausgleich. „Will man die jetzige Krise zum Anlass nehmen, die Europäische Union über die Stabilisierung des Euro hinaus stärker und politisch handlungsfähig zu machen, muss endlich die Diskussion über das Ziel, die Finalité der europäischen Integration geführt und diese Finalité bei den Bürgerinnen und Bürgern Europas verankert werden." Die EU - und das ist hier auch meine These - ,darf nicht länger als technisch-pragmatisches Konstrukt ökonomischer Rationalität mit sich stets steigernder gouvernementaler Dominanz erscheinen, vielmehr muss sie eine politische Ordnungsidee zum Ausdruck bringen, die auf die Völker Europas Bezug nimmt und sie als solche beteiligt. “25

\section{LITERATUR}

Alter, Karen J. (1998): „Who are the ,Masters of the Treaty“?“" in: International Organization 52, 121-147.

Alter, Karen J. (1996): „The European Court's Political Power“, in: West European Politics 19, No. 3, 458-487.

25 Böckenförde 2011, $301 \mathrm{f}$. 
Alter, Karen J./Meunier-Aitsahalia, Sophie (1994): „Judical Politics in the European Community. European Integration and the Pathbreaking Cassis de Dijon Decision“, in: Comparative Political Studies 4, 535-561.

Bast, Jürgen (2010): „Europäische Gesetzgebung - Fünf Stationen in der Verfassungsentwicklung der EU“, in: Franzius, Claudio/ Mayer, Franz/Neyer, Jürgen (Hrsg.), Strukturfragen der Europäischen Union, Baden-Baden: Nomos, 173-180.

Böckenförde, Ernst-Wolfgang (2012): „Kennt die europäische Not kein Gebot?“, in: NZZ-Online. http://www.nzz.ch/nachrichten/kultur/aktu ell/kennt_die_europaeische_not_kein_gebot_1.6182412.html (zitiert: 24.02.2012).

Böckenförde, Ernst-Wolfgang (2011): „Kennt Europas Not kein Gebot?“, in: Böckenförde, Wissenschaft, Politik, Verfassungsgericht, Frankfurt: Suhrkamp, 299-303.

Bogdandy, Armin von (2010): „Prinzipien der Rechtsfortbildung im Europäischen Raum. Überlegungen zum Lissabon-Urteil des Bundesverfassungsgerichts und gegen den methodischen Nationalismus“, in: Franzius, Claudio/Mayer Franz/Neyer, Jürgen (Hrsg.), Strukturfragen der Europäischen Union. Baden-Baden: Nomos, 340-530.

Brandom, Robert B. (2000): Expressive Vernunft, Frankfurt/Main: Suhrkamp, $856 \mathrm{ff}$.

Brunkhorst, Hauke (2012): Legitimationskrisen. Verfassungsprobleme der Weltgesellschaft, Baden-Baden: Nomos, Teil D.

Brunkhorst, Hauke (2011): „Critique of Dualism: Hans Kelsen and the Twentieth Century Revolution of International Law", in: Constellations Vol. 15, 4/2011, 496-512.

Brunkhorst, Hauke (2007): „Unbezähmbare Öffentlichkeit. Europa zwischen transnationaler Klassenherrschaft und egalitärer Konstitutionalisierung“, in: Leviathan 1/07, 12-29.

Brunkhorst, Hauke (2006): „Bologna oder der sanfte Bonapartismus der transnational vereinigten Exekutivgewalten“, in: Zeitschrift für Philosophie und Sozialwissenschaft 1/2006, 1-6. 
Buckel, Sonja/Oberndorfer, Lukas (2009): „Die lange Inkubationszeit des Wettbewerbs der Rechtsordnungen-Eine Genealogie der Rechtsfälle Viking/Laval/Rüffert/Luxemburg aus der Perspektive einer materialistischen Europarechtstheorie“, in: Fischer-Lescano, Andreas/Rödl, Florian/Schmid, Christoph (Hrsg.), Europäische Gesellschaftsverfassung. Zur Konstitutionalisierung sozialer Demokratie in Europa, Baden-Baden: Nomos, 277-296.

Crouch, Colin (2011): Über das befremdliche Überleben des Neoliberalismus, Frankfurt: Suhrkamp.

Dann, Philipp (2002): Looking through the federal lens: the Semiparliamentary Democracy of the EU, Jean-Monnet working paper 5.

Fisahn, Andreas (2011): „Europa braucht einen neuen Gesellschaftsvertrag“, in: Vorgänge 4/2011, 48-60.

Fischer-Lescano, Andreas/Teubner, Gunther (2006): Regime-Kollisionen, Frankfurt: Suhrkamp.

Fossum, John Erik/Menéndez, Augustín José (2011): The Constitution's Gift. A Constitutional Theory for a Democratic European Union, Plymouth: Rowman.

Gaitanides, Charlotte (2005): „Die Verfassung für Europa und das Europäische System der Zentralbanken“, in: Gaitanides, Charlotte/ Kadelbach, Stefan/Rodriguez Iglesias, Gil Carlos (Hrsg.), Europa und seine Verfassung, Festschrift Zuleeg, Baden-Baden: Nomos, $550-558$.

Habermas, Jürgen (2011): Zur Verfassung Europas. Ein Essay, Frankfurt: Suhrkamp.

Habermas, Jürgen (1992): Faktizität und Geltung, Frankfurt: Suhrkamp.

Halberstam, Daniel/Möllers, Christoph (2009): „The German Constitutional Court says Ja zu Deutschland“, in: German Law Review 8/2009.

Hardt, Michael/Negri, Antonio (2003): Empire. Die neue Weltordnung, Frankfurt: Campus. 
Hitzel-Cassagnes, Tanja (2011): Entgrenzung des Verfassungsbegriffs. Eine institutionentheoretische Rekonstruktion, Baden-Baden: Nomos.

Holmes, Pablo (2012): Verfassungsevolution in der Weltgesellschaft. Differenzierungsprobleme des Rechts und der Politik (Dissertation, Universität Flensburg), Baden-Baden: Nomos (Reihe Politische Soziologie).

Joerges, Christian (2003): „Europe a Großraum? Shifting Legal Conceptualisations of the Integration Project", in: Joerges, Christian/ Ghaleigh, Navraj S. (Hrsg.), Darker Legacies of Law in Europe: The Shadow of National Socialism and Fascism over Europe and its Legal Traditions, Oxford: Hart Publishing, 167-191.

Judt, Tony (2010): Ill Fares the Land, New York: Penguin.

Kemmerer, Alexandra (2010): „Legitimationssubjekte: Staatsbürger und Unionsbürger“, in: Franzius, Claudio/Mayer, Franz C./Neyer, Jürgen (Hrsg.), Strukturfragen der Europäischen Union.

Ley, Isabelle (2010): „Brünn betreibt die Parlamentarisierung des Primärrechts. Anmerkungen zum zweiten Urteil des tschechischen Verfassungsgerichtshofs zum Vertrag von Lissabon vom 3.11.2009“, in: Juristen-Zeitung 65 (4), 170.

Marks, Susan (2000): The Riddle of all Constitutions, Oxford: Oxford Univ. Press.

Marx, Karl (1985): Der 18. Brumaire des Louis Bonaparte, MEGA I/11, Berlin: Dietz.

Möllers, Christoph (2005): „Transnationale Behördenkooperation. Verfassungs- und völkerrechtliche Probleme transnationaler administrativer Standardsetzung“, in: Zeitschrift für ausländisches öffentliches Recht und Völkerrecht 65, 351-389.

Möllers, Christoph (2003): Gewaltengliederung, Habilitationsschrift, Heidelberg.

Prien, Thore (2010): Fragmentierte Volkssouveränität, Baden-Baden: Nomos.

Schönberger, Christoph (2009): „Lisbon in Karlsruhe: Maastricht's Epigones at Sea“, in: German Law Review 8/2009. 
Schönberger, Christoph (2005): Unionsbürger. Europas föderales Bürgerrecht in vergleichender Sicht, Tübingen: Mohr Siebeck.

Somek, Alexander (2008): Individualism: An Essay on the Authority of the European Union, Oxford: University Press.

Streek, Wolfgang (2010): „Noch so ein Sieg, und wir sind verloren. Der Nationalstaat nach der Finanzkrise“, Leviathan 38: 159-173

Thiele, Ulrich (2011): „Von der Volkssouveränität zum Völker(staats)recht“, in: Eberl, Oliver (Hrsg.), Festschrift für Ingeborg Maus, 175-196.

Tuori, Kaarlo/Sankari, Suvi (Hrsg., 2010): The Many Constitutions of Europe. Oxon: Ashgate.

van Ooyen, Robert (2010): Zum Europasyndrom des Verfassungsgerichts: Die Staatstheorie des Bundesverfassungsgerichts und Europa. Von Solange über Maastricht zu Lissabon, 3. Aufl., BadenBaden: Nomos.

Wegmann, Milène (2010): „European Competition Law: Catalyst of Integration and Convergence", in: Tuori, Kaarlo/Sankari, Suvi (Hrsg.), The Many Constitutions of Europe, Oxon: Ashgate, 91-107. 